\title{
Arthrogryposis multiplex congenita Part 2: Muscle pathology and pathogenesis
}

\author{
DARAB K. DASTUR, ZOHRA A. RAZZAK, AND E. P. BHARUCHA \\ From the Neuropathology Unit, J.J. Group of Hospitals, and \\ the Children's Orthopaedic Hospital, Bombay, India
}

SUMMARY Pathological findings are reported on 34 specimens from 16 cases of arthrogryposis multiplex congenita (AMC), including initial observations on paraffin sections from 28 muscles, and subsequent observations on six additional specimens from three of these cases studied both histologically and histochemically. Thirteen of the 34 specimens (from 11 cases) were histologically normal, probably on account of an unaffected muscle being sampled. The most constant pathological feature in the remaining specimens was a disorganization of the muscle fibres and fascicles by severe fibrosis; only three specimens (from two cases) did not show this. Very thin faintly striated muscle fibres embedded in this matrix were encountered in 10 specimens from nine cases. An attempt at grouping of these atrophic or ill-developed fibres was noticed in four specimens; but this may not be denervation atrophy. Two specimens (from two cases) showed 'myopathic' features. Repeat biopsy after two to three years was carried out on two affected muscles each from three patients. Case 3 showed well preserved but uniformly small fibres. Case 4 showed extremely few and scattered small rounded fibres. Case 14 showed pronounced variation of fibre size in both, with both atrophic and hypertrophied fibres. Normal nerves and spindles were seen in all these six specimens irrespective of the state of the muscle, and excessive fibro-fatty tissue in cases 4 and 14. Histochemical examination for oxidative enzymes, ATPase, and phosphorylase in these six specimens revealed a normal checkerboard pattern and ratio of type I and type II fibres, in case 3 only. The muscles of case 4 showed a preponderance of type I fibres. One specimen from case 14, showed the same fibres reacting for both oxidative enzymes and phosphorylase, suggesting a lack of development of fibres. The intrafusal fibres were mainly of type $I$ in all. Two possible pathogenetic mechanisms operating in early embryonic life, which may lead to the characteristic changes of AMC, are discussed: (1) a defect in the development of the muscle whereby the full recruitment of myoblasts from the mesenchyme of the limb-bud does not take place and muscles do not form adequately; (2) a lack of innervation of the muscles on account of arrested growth of anterior horn cells. The combined operation of both these mechanisms is also considered. Fibrous tissue replaces the muscle tissue that is lacking, and contractures and deformities ensue. The evidence gathered on our material, such as the very thin smooth muscle fibres, the large numbers of well-formed nerves and spindles especially in the repeat biopsies, and the above-mentioned histochemical feature, would appear to favour the hypothesis of ill-developed muscles in the production of AMC in the majority; in the rest denervation playing either a major or concurrent role.

A brief review of the literature implicating the joints and the central nervous system in the production of arthrogryposis multiplex congenita (AMC), has already been presented (Bharucha et al., 1972). Changes in the muscle have also been held responsible for the deformities.

In 1908, Howard described the condition as 'dystrophia muscularis congenita', emphasizing the muscular part of the defect. Middleton (1934) observed excessive amounts of fat and connective tissue in muscle biopsies and therefore emphasized the myopathic nature of the condition, which he called 'myodystrophia foetalis deformans'. Rossi (1947) recognizing the condition to be a clinical syndrome with joint and muscle involvement called it 'arthro-myodysplastic syndrome'. Banker, Victor, and Adams (1957) reported two cases of 'arthrogryposis multiplex due to congenital muscular dystrophy', 
where the deformities were considered secondary to the muscle changes, which were 'identical to those seen in progressive muscular dystrophy'. Subsequently Pearson and Fowler (1963) reviewed eight cases (two of their own in sibs) of muscular dystrophy with probable arthrogryposis.

Sheldon (1932) had introduced the concept of muscular maldevelopment, through the nomenclature 'amyoplasia congenita'. He postulated an aplasia, hypoplasia, or dysplasia of certain muscle groups leading to an imbalance at joints. Hillman and Johnson (1952) also suggested muscular aplasia as the primary lesion in arthrogryposis multiplex congenita. Kite (1955) had further incriminated a failure of embryonic myoblasts as the first cause of all the changes. Frischknecht, Bianchi, and Pilleri (1960) reported cerebral defects, degeneration of pyramidal tracts and anterior horn cells, and pseudomyopathic changes in muscles in a family with arthrogryposis which he described under the all-inclusive title of "neuro-arthro-myo-dysplasia congenita'. Swinyard and Mayer (1963) reviewed 70 cases and felt that the primary pathological change may be in the spinal cord, in the muscles themselves, or in the connective tissue. They expressed uncertainty about AMC being either a 'single disease entity' or a 'manifestation of several different aetiologies'.

To the best of our knowledge, there is only one recent histochemical study of the muscles in AMC. Fenichel (1969) in a study of cerebral influence on muscle fibre typing includes seven cases with 'arthrogryposis', according to the definition of this condition given by Drachman and Coulombre (1962) that it is 'a non-specific sign of foetal immobilization'. Fenichel considers 'arthrogryposis' to be a 'model of skeletal fixation during muscle maturation due to abnormal suprasegmental function'.

The main objectives of this study have been described by Bharucha, Pandya, and Dastur (1972). In the present paper the histological changes observed in the muscles will be described and our current views on the pathogenesis of AMC will be presented.

\section{METHODS}

Initially 28 muscle biopsy specimens were available from 14 cases (two specimens each were available from 10 cases and three from two). Paraffin sections stained by haematoxylin and eosin ( $\mathrm{H}$ and $\mathrm{E})$, the picro-Mallory method for connective tissue, and by phosphotungstic acid haematoxylin (PTAH) were examined in each case. Muscle fibre diameters were measured in most by micrometry.

Later, from three of the children who could be recalled, two further muscle biopsy specimens from each could be obtained. Fresh frozen sections of these were processed for the following histochemical reactions: succinic dehydrogenase (SDH) and reduced nicotinamide adenine dinucleotide (NADH), for oxidative enzyme activity; myofibrillar adenosine triphosphatase (ATPase); and phosphorylase for glycolytic enzyme activity. Fibre diameters were measured and ratios of type I to type II fibres were obtained. In addition, fixed frozen and paraffin sections were stained with $\mathrm{H}$ and $\mathrm{E}$, picro-Mallory, and PTAH stains. Thus in all 34 muscle biopsies were studied.

Serum creatine phosphokinase (CPK) was estimated in four cases by the method of Hughes (1962).

\section{RESULTS}

FIRST EXAMINATION The histopathological observations in all cases biopsied are summarized in Table 1. The largest pathological group of

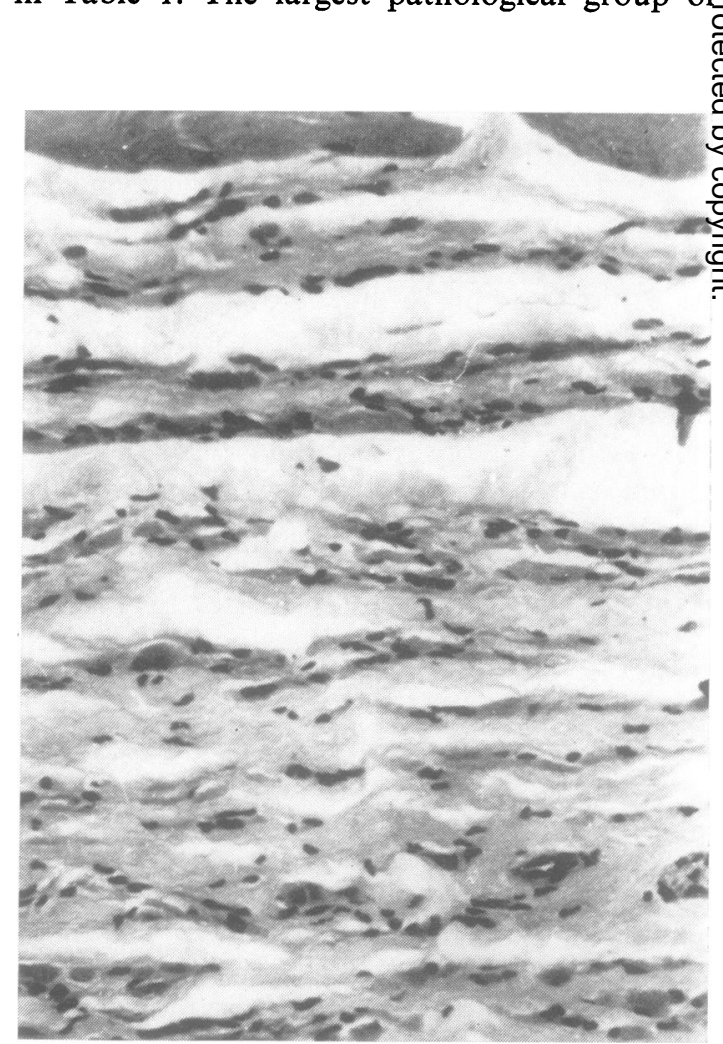

FIG. 1. $(N P / E / 830 b)$. Very thin muscle fibres embedded in fibrous tissue; two normal sized fibres at the upper end. $H$ and $E, \times 265$. 
(a)

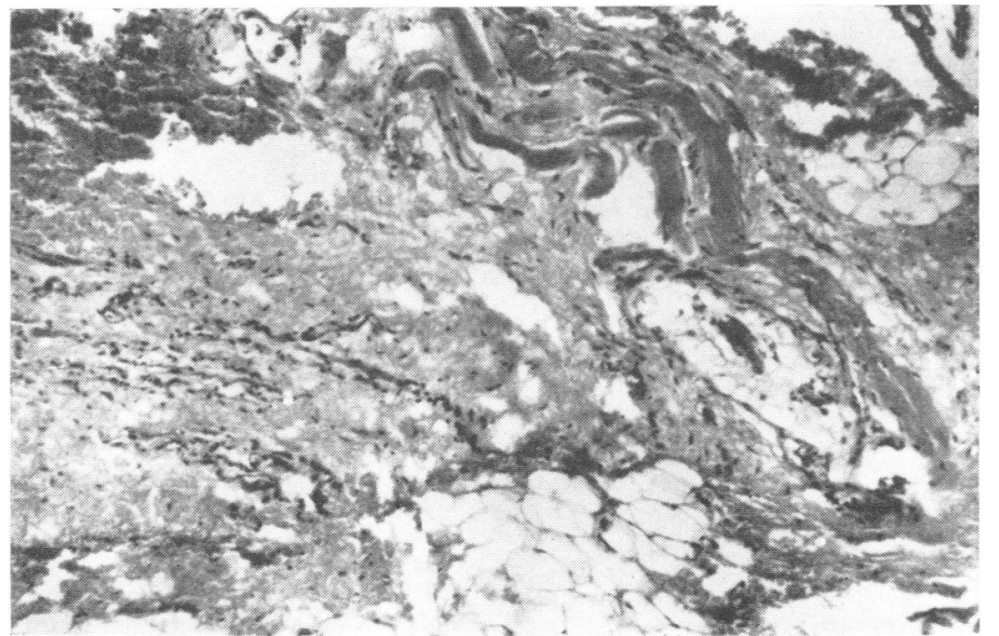

FIG. 2. (NP/E/613a). (a) IIldefined group of very thin muscle fibres contrasting with group of well-formed fibres, both in fibrous matrix. Note essentially normal muscle spindle in the latter. $H$ and $E$, $\times$ 105. (b) Same muscle showing cross-striations on very thin fibres of slightly varying size PTAH, $\times 660$.
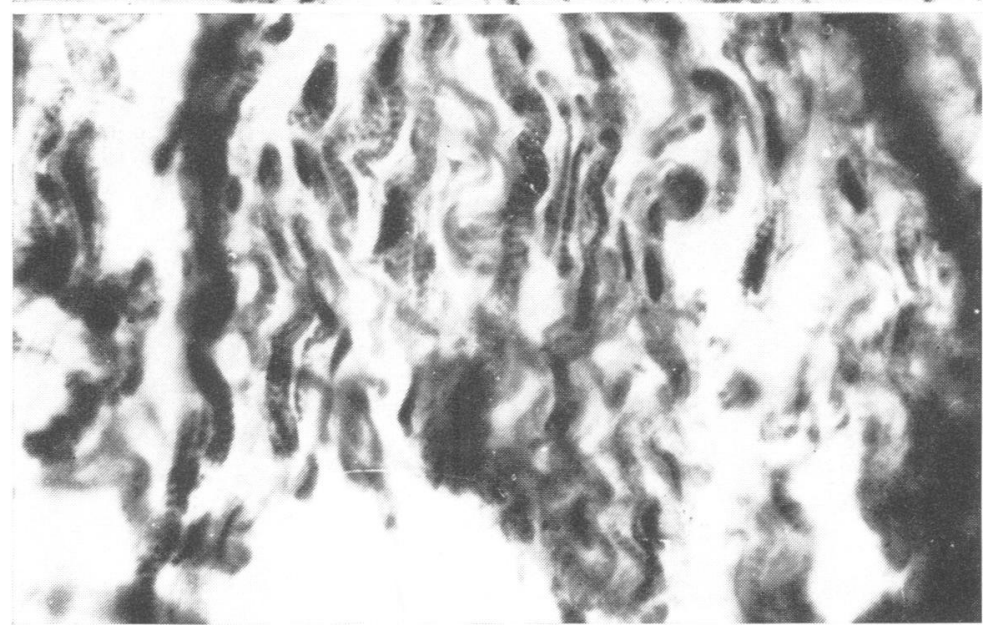

muscle comprising 10 specimens from nine cases, showed a similar type of change, of greater or lesser severity in different cases. This consisted essentially of a dense fibrosis with total lack of organization of muscle into fascicles together with the presence of very fine muscle fibres embedded in this fibrous tissue. Thus Fig. 1 shows very fine strands of muscle fibres in a collagenous matrix, contrasting strikingly with two much larger normal fibres. In another area of the same muscle, fibres of a slightly larger dimension, of varying sizes but still small, are seen. This was from the quadriceps muscle of a 2 year old girl, with an average fibre diameter of $9.7 \mu$ only and a range of 5-17 $\mu$. In this case the biopsy of another muscle, the deltoid, showed even less muscle tissue in the form of thin straggly fibres.
A clearer contrast between the better preserved and the thin fibres which are now attempting a grouping is seen in Fig. 2a from the deltoid muscle of an infant aged 15 months; a normal muscle spindle was also encountered. Even the very small fibres which average $9.4 \mu$ in size, in contrast to the normal fibres of $38 \cdot 1 \mu$, showed clear cross-striations with PTAH staining (Fig. $2 b$ ). In five muscle specimens from five cases PTAH stain revealed cross-striations even in thin fibres in which $\mathrm{H}$ and $\mathrm{E}$ failed to reveal the same. A picture more clearly indicative of group atrophy (Fig. 3a) is seen at times, as in the specimen of biceps muscle of a 4 year old boy. Closer examination of such fibres, in $\mathrm{H}$ and $\mathrm{E}$ stained sections, generally showed them to be smooth and with crowding of nuclei on the fibres (Fig. 3b). Essentially normal muscle 


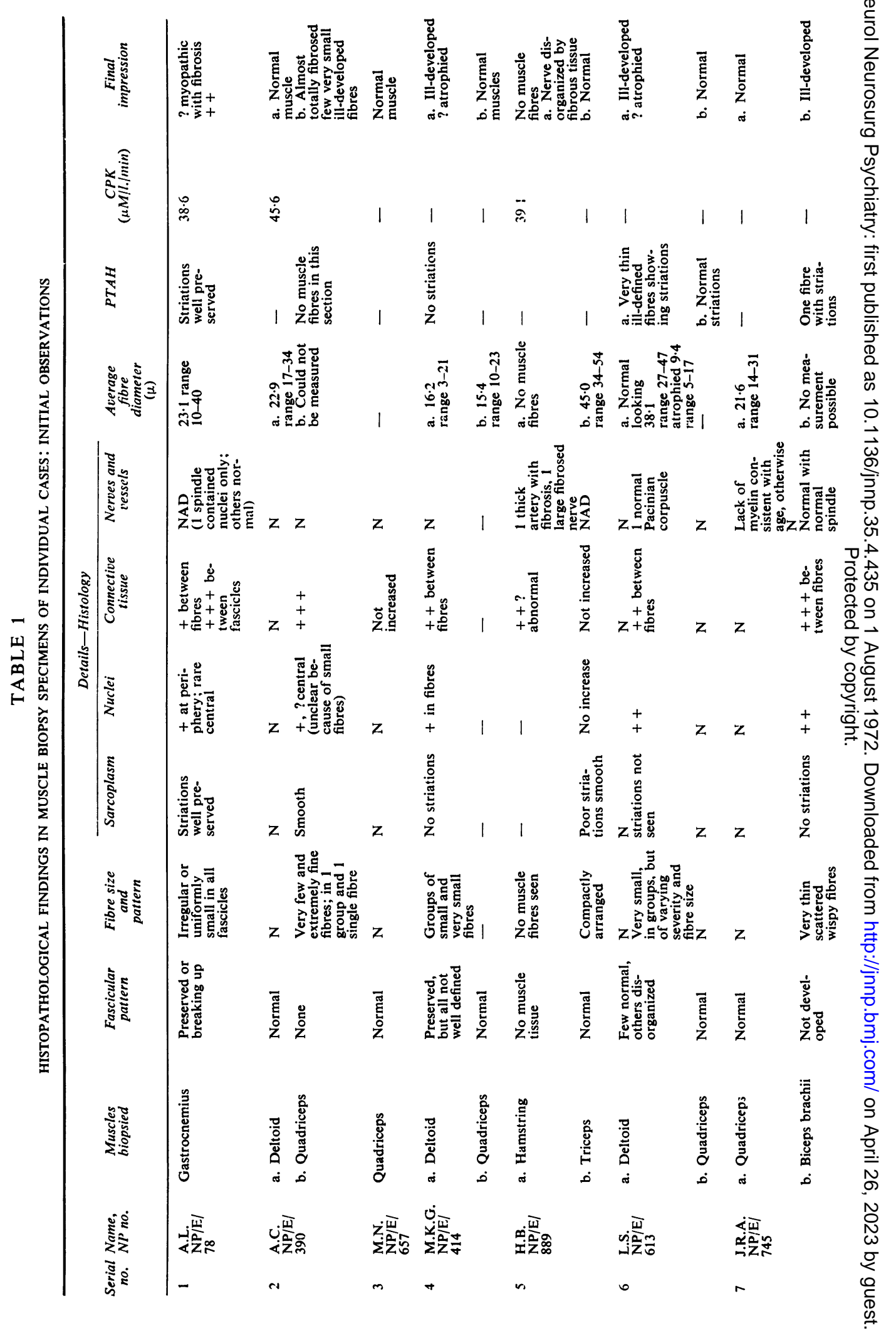




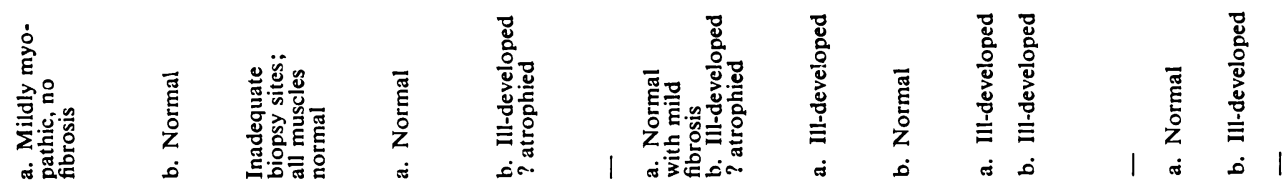

$\stackrel{\leftrightarrow}{m}$

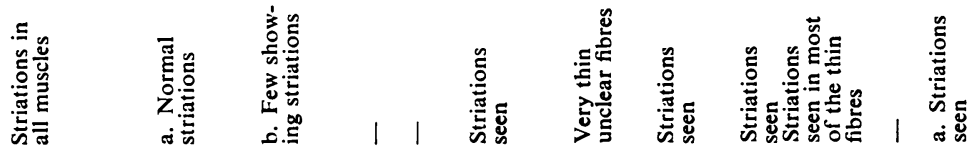

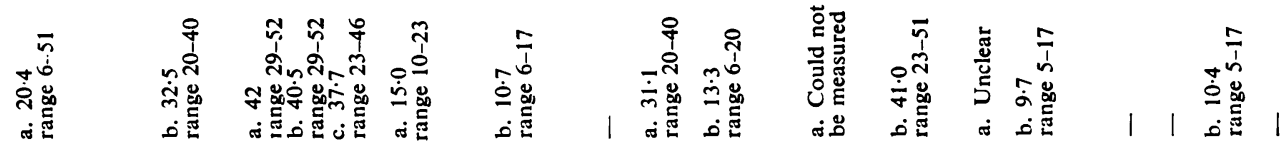

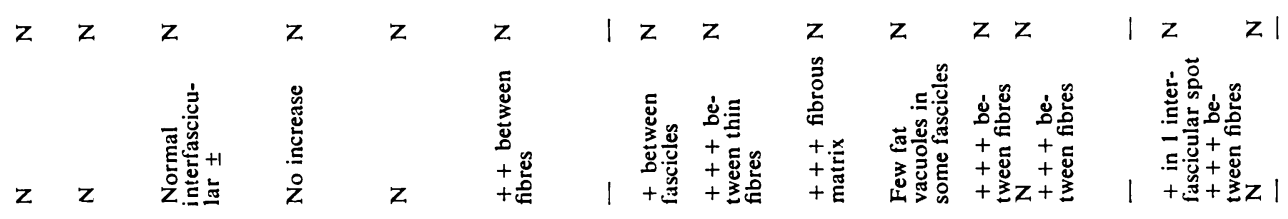

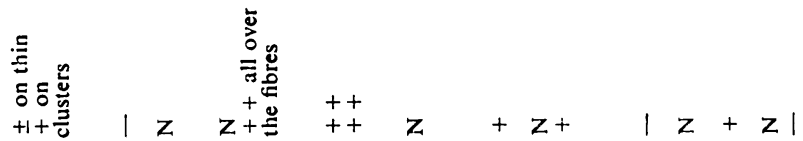

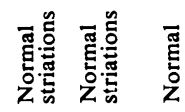

党

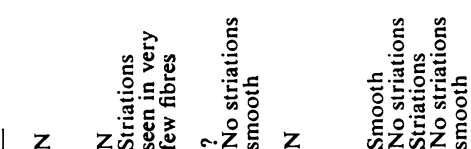

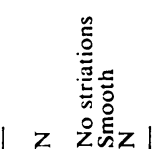

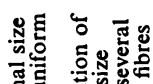

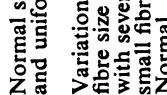

z

(

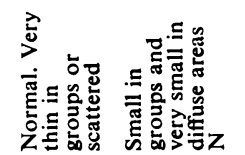

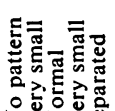

$\mathrm{z}$ 胥

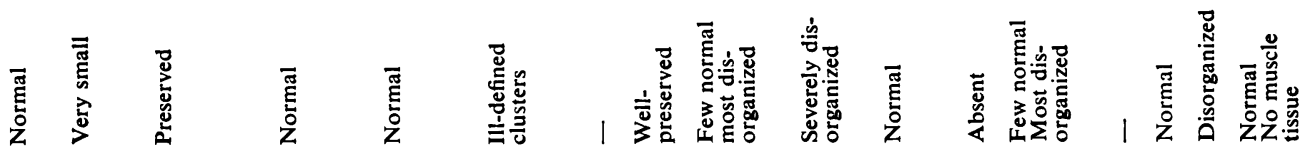

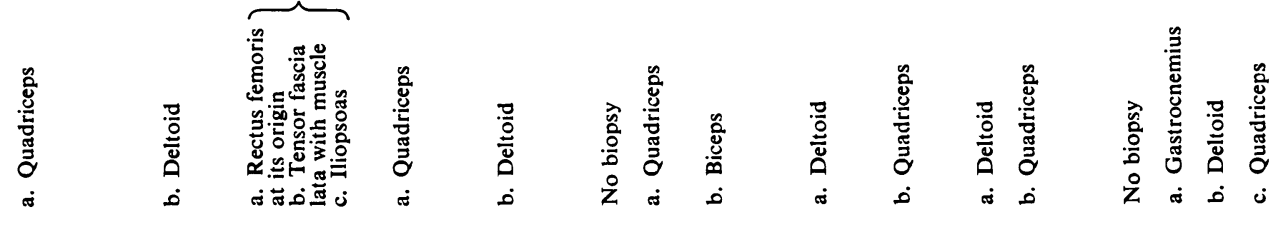

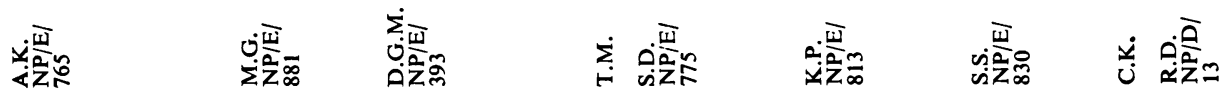

은

$=\simeq$

m

$\pm$

$\simeq \underline{0}$ 
(a)

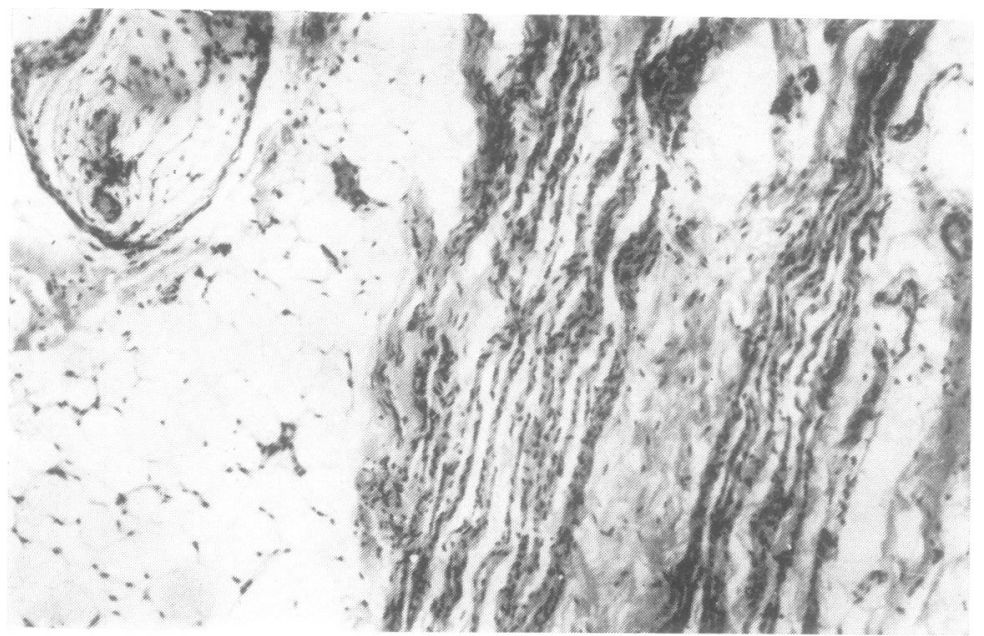

(b)

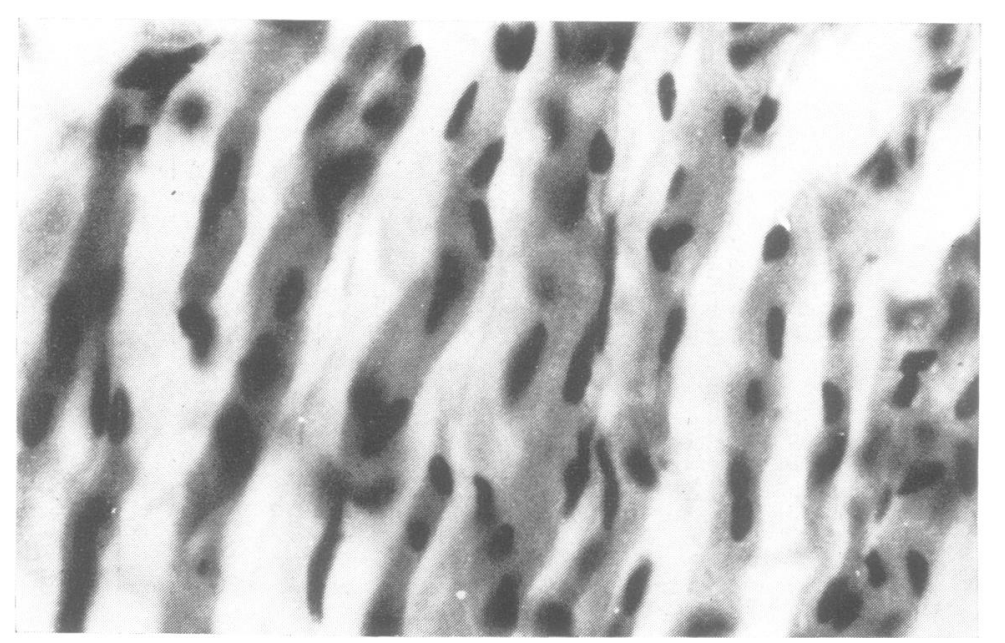

FIG. 3. $(N P / E / 775 b)$. (a) $T$ wo well-defined groups of thin muscle fibres in fibro-fatty tissue; note muscle spindle with prominent connective tissue and intrafusal fibres. (b) Closer view. of some of these muscle fibres showing smooth appearance and $\stackrel{\mathrm{\omega}}{\mathrm{S}}$ dark proliferated nuclei. Both $H$ and $E$, (a) $\times 105$, (b) $\times 660$.

\section{క్}

FIG. 4. (NP/E/390, 2). Solitary small group of very tiny muscle fibres in a section otherwise totally devoid of muscle tissue. $H$ and $E, \times 660$.

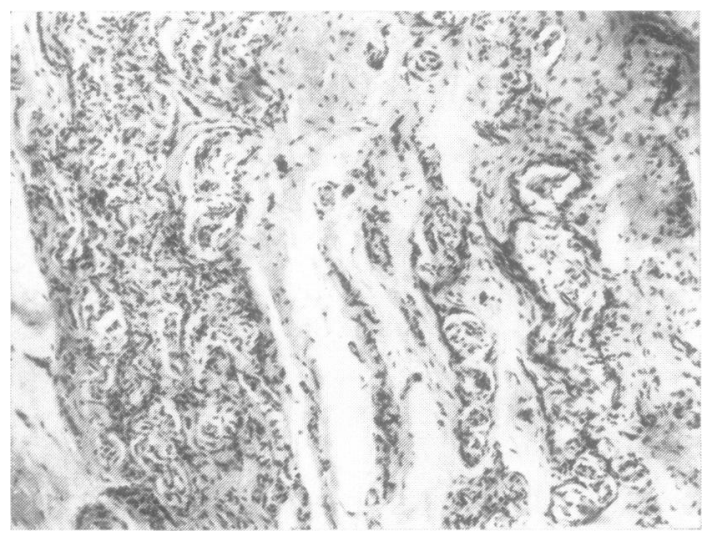

FIG. 5. $(N P / E / 889,1)$. Degenerating intramuscular $N$ nerves with proliferated sheath nuclei, embedded in $N$ fibrous tissue. $H$ and $E, \times 105$. 


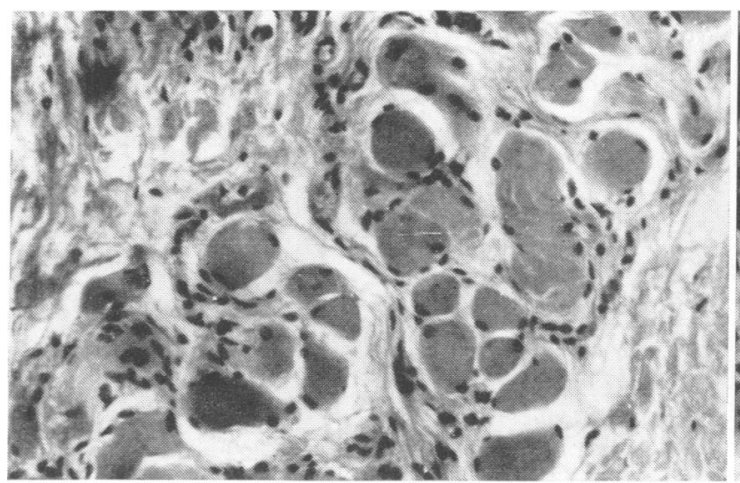

(a)

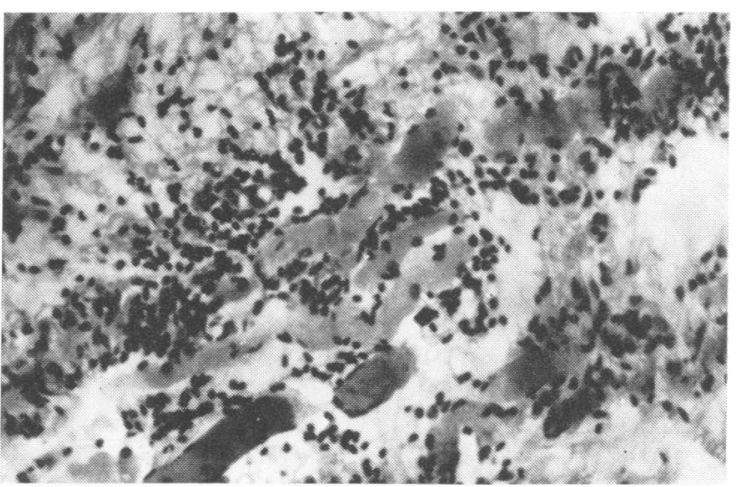

(b)

FIG. 6. (NP/E/78). Case of 'myopathic' AMC. (a) Endo- and perimysial increase of connective tissue, rounded fibres cut in T.S. (b) Area of small mononuclear cell infiltration among muscle fibres separated by fibro-fatty tissue. Both $H$ and $E, \times 265$.

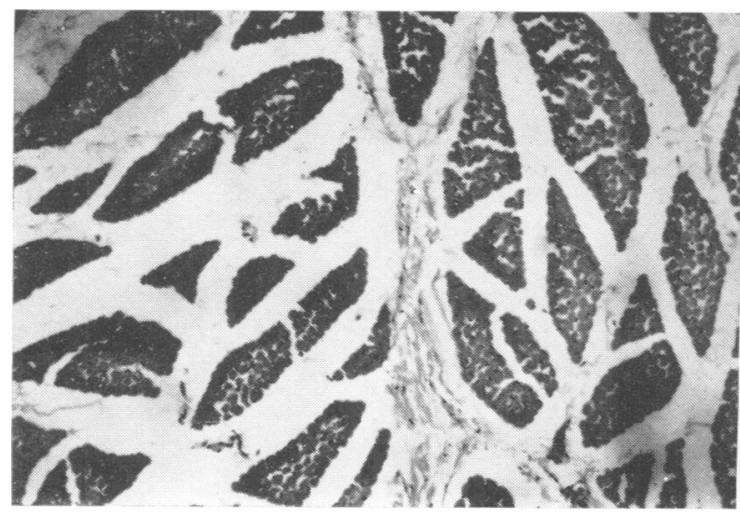

(a)

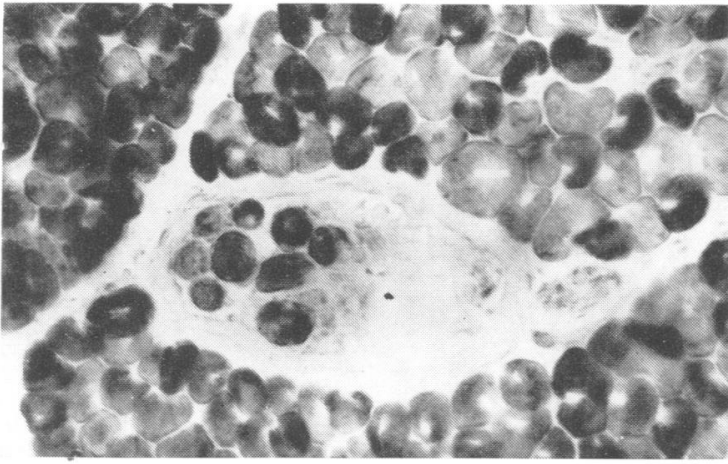

(c)

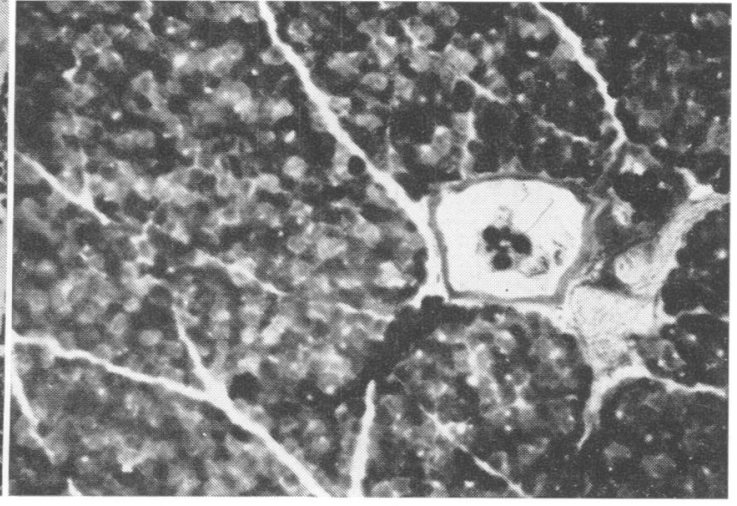

(b)

FIG. 7. (NP/G/39a). Right biceps brachii muscle. (a) Small fascicles made up of uniformly atrophied fibres. $H$ and $E, \times 105$. (b) Normal checkerboard pattern of type I and type II extrafusal fibres. Intense activity of intrafusal fibres in tandem spindles. $\mathrm{NADH}_{2}, \times 105$. (c) Intrafusal fibres showing varying grades of activity; nerves not showing activity. Normal checkerboard pattern of extrafusal fibres. Phosphorylase, $\times 265$. (d) Intense activity in the two nerve twigs, probably in the Schwann sheath. ATPase, $\times 265$.

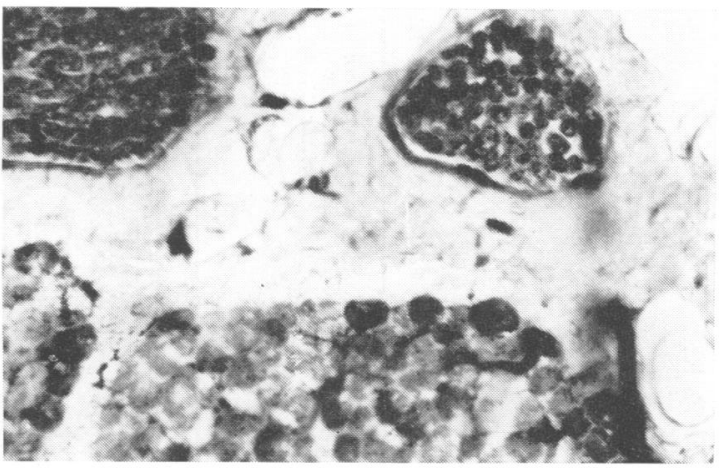

(d) 


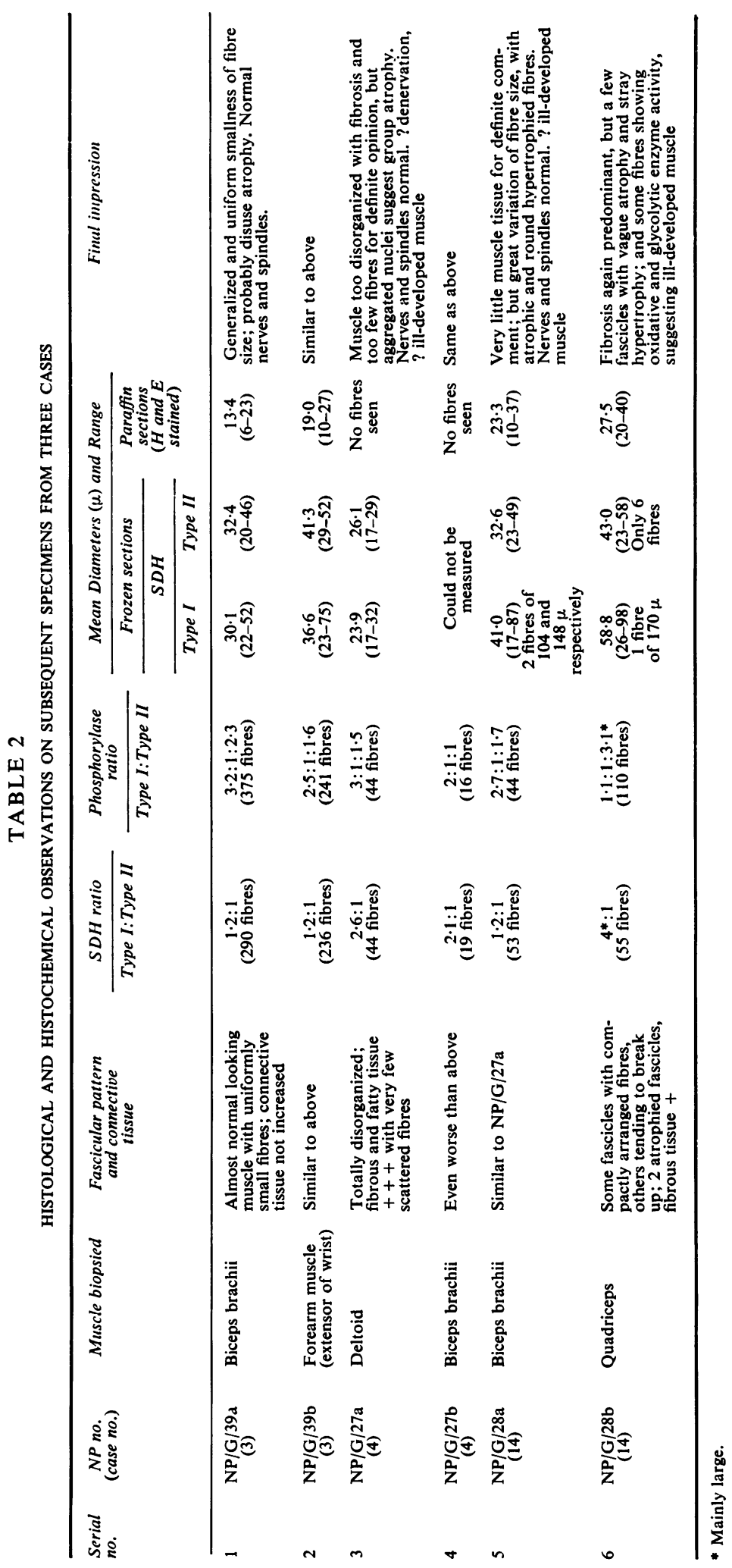




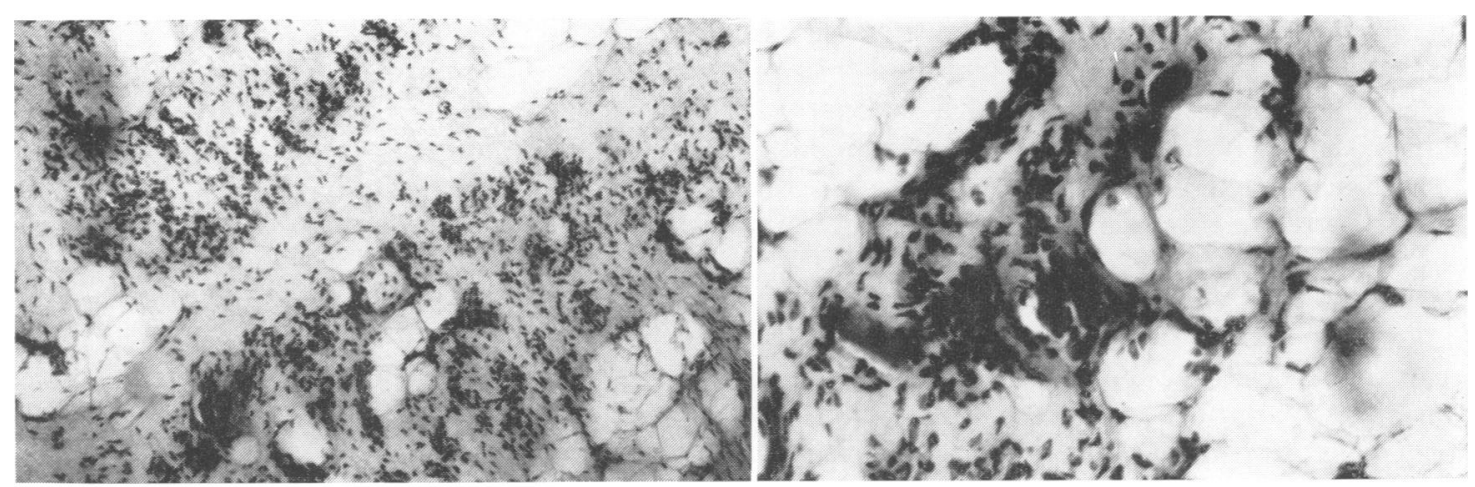

(a)

(b)

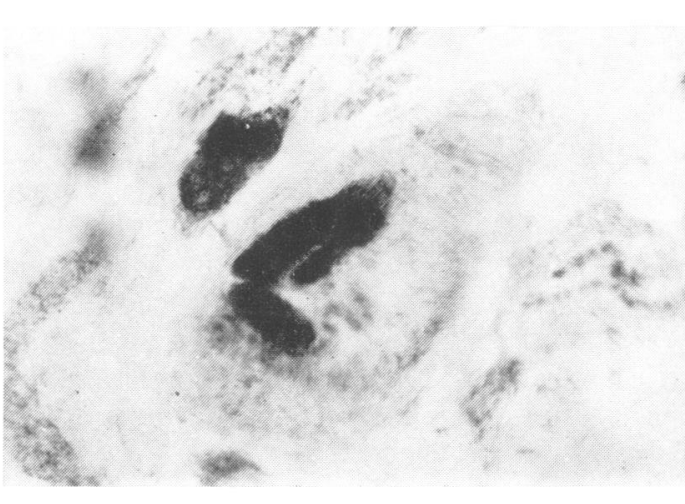

(c)

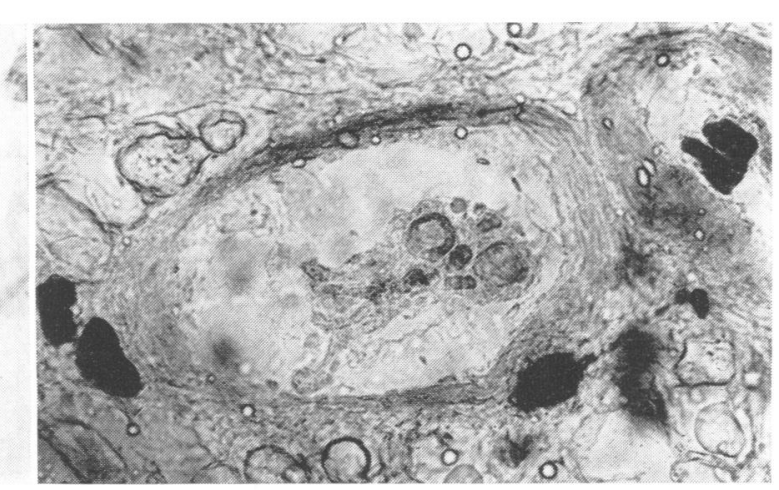

(d)

FIG. 8. (NP/G/27a). Right deltoid muscle. (a) Clustered nuclei in two large groups suggesting fascicular outline. (b) Closer view of some of the clusters showing stray small muscle fibres in them. Both frozen sections, $H$ and $E, \times 105$ and $\times 265$ respectively. (c) Strongly type I intrafusal fibres and one or two small extrafusal fibres; nerve approaching spindle and within it is segmentally stained. SDH, $\times 265$. (d) Strongly positive type II fibres, extrafusal and intrafusal in one spindle, the other larger spindle showing type I fibres, which were unstained but brought to view by reducing the illumination. Phosphorylase, $\times 265$.

spindles are seen in such cases without atrophy of the intrafusal fibres-for example, Figs $2 \mathrm{a}$ and $3 \mathrm{a}-$ with occasionally slight prominence of intrafusal connective tissue (Fig. 3a).

At times, the fibrosis or the lack of differentiation into muscle tissue were so pronounced that only the presence of a muscle spindle indicated that the biopsy was in fact from a muscle. At other times, search through an entire section would reveal only an extremely small group of muscle fibres in a specimen otherwise made up of fibrous tissue only, as in Fig. 4 from a quadriceps muscle of an 8 month old girl. Here the fibre diameter could not be measured.

Only one of these 34 specimens of muscle, including those belonging to this group of 10 , showed a similar fibrosing and disorganizing change in an intramuscular nerve (Fig. 5). Sheath cell proliferation accompanied the myelin loss in this nerve twig. In this specimen of a hamstring muscle from an 11 year old girl, no muscle tissue was to be seen at all. Another muscle of this case was normal.

In all, 13 of the initial 28 biopsied muscles were histologically normal. These were generally clinically less affected or unaffected muscles biopsied for contrast with the more affected muscle. In case 9, however, all three biopsy specimens were normal on account of their being restricted to the site of surgical exposure which was the region of the congenitally dislocated hip joint.

In none of the four cases so tested was there any elevation of the serum creatine phospho- 
kinase (CPK) level, including the two cases where clinical, electromyographic, and histological evidence was in favour of a myopathic change. Thus in case 8 there was a mildly dystrophic change in the form of variation of muscle fibre size (range 6-61 $\mu$ ) though without an increase in connective tissue. The other muscle in this case did not show even this change. The other myopathic patient in our series (case 1) showed a more characteristic change of variation in size and rounding of fibres together with increase of fibrous and fatty tissue and some accumulations of small mononuclear cells (Figs 6a, b). The less affected child (case 8 ) had an older sib similarly affected. The other child (case 1) showed the more florid myopathic change in the gastrocnemius muscle, which, however, was not enlarged. This child also had the 'policeman-tip' position of the extremities, characteristic of AMC.

In two of the specimens there was no muscle tissue at all and it was difficult to say whether this was consequent to the arthrogrypotic changes or mainly the result of an inadequate biopsy. Both these cases however showed histological evidence in their other muscle biopsy of fibrosed nerves (case 5) and ill-developed fibrosed muscle (case 16) respectively.

SUBSEQUENT EXAMINATION From three of the patients (nos. $3,4,14$ ) who could be recalled for a second examination two to three years after the first, two muscle biopsies each could be obtained.

In one of them (case 3) with more flexion contracture at elbows than at knees, where originally a normal quadriceps muscle had been sampled, weak muscles of the arm and the forearm were now biopsied. The biceps brachii and an extensor muscle from a forearm both showed generalized smallness of fibre-size (Table 2), without group atrophy. $\mathrm{H}$ and $\mathrm{E}$ stained frozen sections showed this clearly (Fig. 7a). Histochemical preparations confirmed this finding in addition to revealing a normal checkerboard pattern of type I and type II fibres. Both the oxidative enzymes, SDH and NADH, demonstrated this (Fig. 7b), as also the intense positivity of intrafusal fibres. The roughly equal proportion of type I and type II fibres in both muscles (Table 2) was observed with both oxidative enzymes and phosphorylase (Fig. 7c). The latter also demonstrates that occasional spindles contained type II intrafusal ô fibres. While nerves were faintly stained with those enzymes (Fig. 7b, c, where nerves are seen outside the spindle), they showed a more intense activity for ATPase (Fig. 7d) where the Schwann cell outline is revealed, possibly by the 0 mitochondrial ATPase (Padykula and Herman, 1955). The spindle capsule and nuclei have also taken up the stain.

Case 4 who had originally presented with a clearly neuropathic picture on EMG and changes of dubious nature in one deltoid muscle at follow-up showed severe disorganization and lack of muscle tissue in both deltoid and biceps muscles of the other limb. There were only rare small fibres encountered in a fibro-fatty matrix. An interesting feature was the presence of $\overrightarrow{0}$ clusters of nuclei arranged in a peculiar fashion, as though representing the totally atrophied fibres within a fascicle. This feature is clearly seen in Figs 8a, b, where two such clusters show two or three fibres remaining. Both the deltoid of and biceps muscles were similar to each othero $f$ the latter showing even fewer fibres and no्ष nuclear clusters. There was a preponderance o웅을 type I fibres in all the enzyme preparation ${ }_{-}$ (Table 2).

Over large areas there was no recognizable 0 muscle tissue, but only muscle spindles. Whili o all spindles in preparations for oxidative enzyme $\overrightarrow{0}$ activity contained type I intrafusal fibres onl $\vec{y} \vec{N}$ (Fig. 8c), the spindles encountered in the preparations processed for phosphorylase activity showed type I and/or type II intrafusal fibres-for example, Fig. 8d. The nerve fibres outside and within the spindles showed only slight positive activity (Fig. 8c).

One of the muscles (biceps brachii) from the third patient with neurogenic atrophy (case 14) diagnosed on original EMG examination, showed severe disorganization very similar to that in case 4 , except that there were comparatively more fibres with obvious variation of fibre size (Table 2). In one field, type I fibres 20 , $37,78,104$, and $148 \mu$ in diameter were encountered (Figs 9a, b). In muscle specimens of adult control subjects we have noted the mean fibre diameter of type I fibres to be $78 \pm 17 \mu$; truly atrophic fibres were considered to be those less than $44 \mu$ or two standard deviations (SDs) below the mean and truly hypertrophic fibres to be more than $114 \mu$, two SDs above the mean (Razzak, 1970). Considering that we are dealing here with a child aged about 5 years there is 


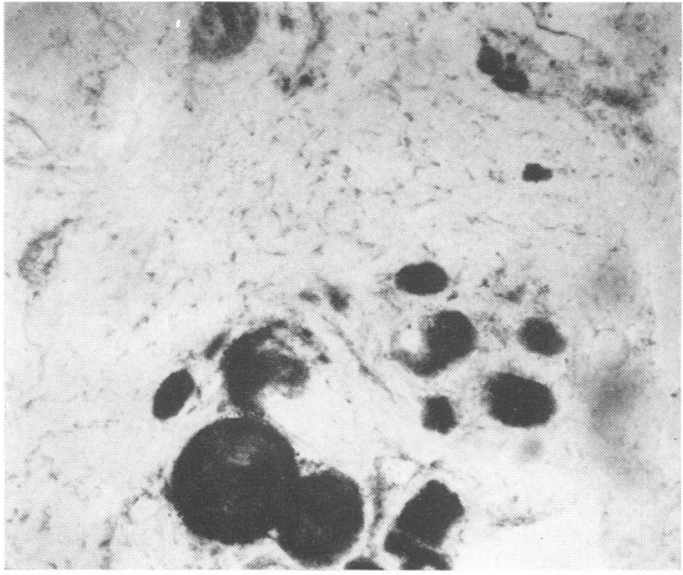

(a)

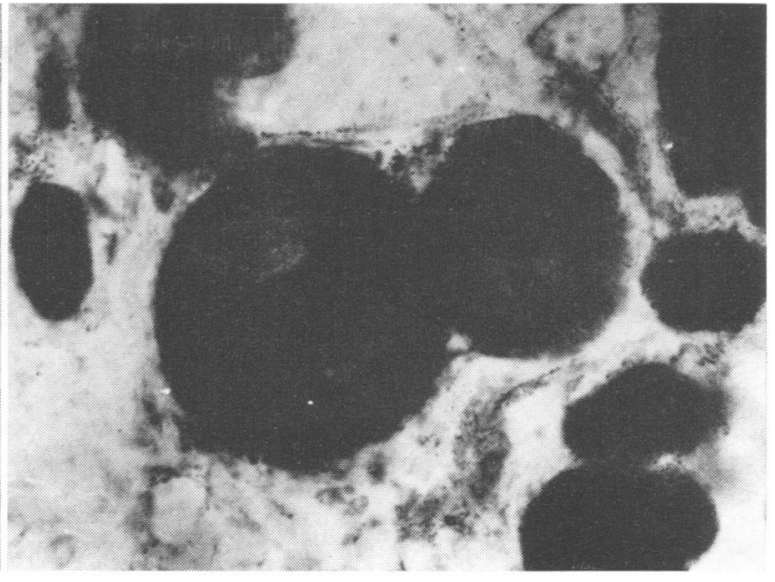

(b)

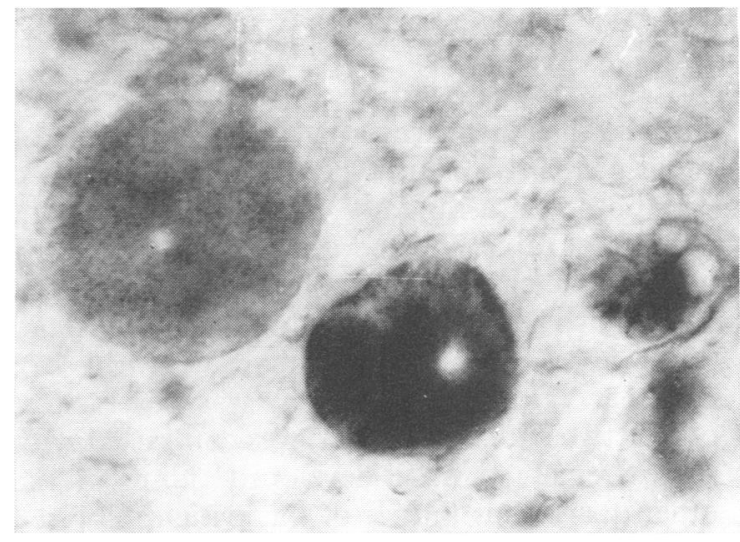

(c)

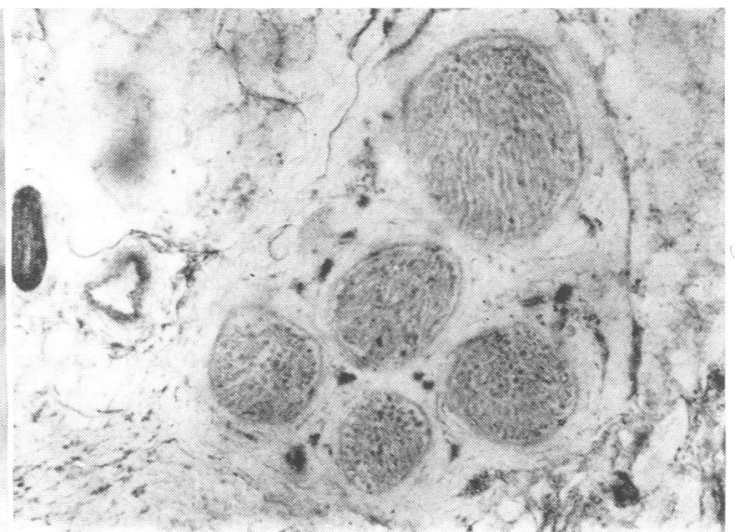

(d)

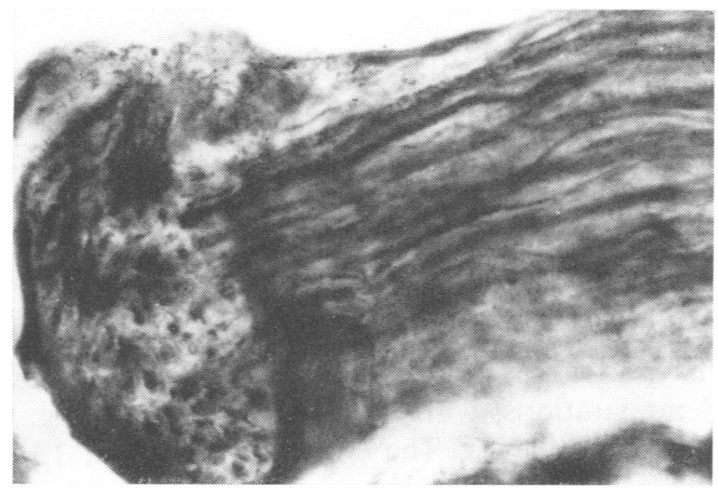

(e)

FIG. 9. (NP/G/28a). Right biceps muscle. (a) Scattered type I fibres of varying size in a loose fibrous matrix. (b) Closer view of some of the fibres, which were actually measured, including one hypertrophied fibre (see text). (Both SDH, $\times 105$ and $\times 265$ respectively.) (c) Two type II and one intermediate type scattered fibres, one of the former showing vacuolation. Phosphorylase, $\times 660$. (d) Faintly defined nerve fibres in five bundles of a large intramuscular nerve. SDH, $\times 105$. (e) Same nerve showing stronger activity in axon and weaker in fibre sheaths. $\mathrm{NADH}_{2}, \times 660$. 


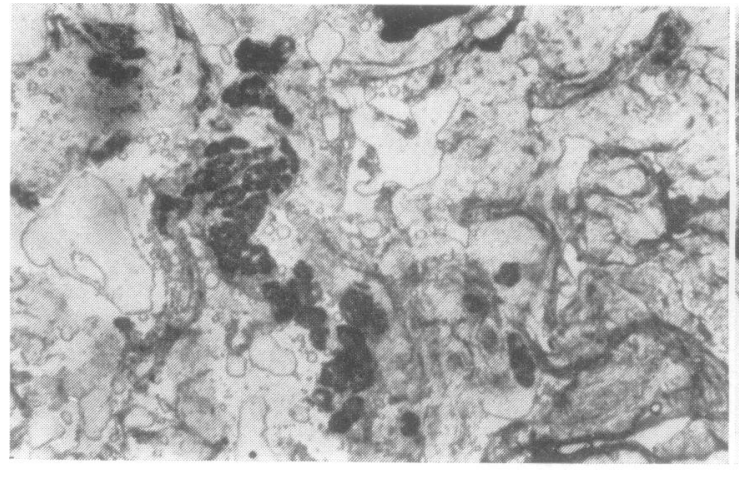

(a)

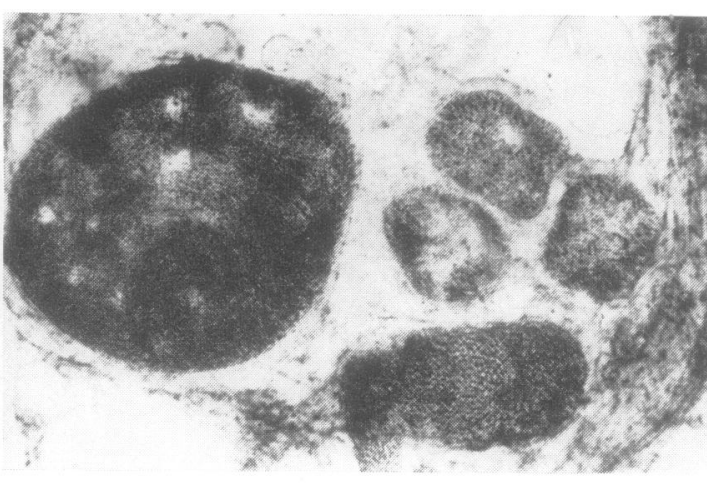

(b)

FIG. 10. (NP/G/28b). Quadriceps muscle. (a) Vaguely defined group of muscle fibres of varying size and uniform activity in fibro-fatty matrix. (b) Another group showing four medium-sized and one excessively hypertrophied fibre (see text). Both SDH, $\times 40$ and $\times 265$ respectively.

true atrophy as well as true hypertrophy of her muscle fibres.

Rarely, there were small fibres with vacuoles, appearing as degenerating muscle fibres, as seen in the type II fibres in the phosphorylase preparation shown in Fig. 9c. The largest fibre seen in this Figure is possibly of intermediate type. The phosphorylase reaction, like the ATPase, appeared to reveal a small but ever present proportion of fibres of a type intermediate between types I and II. This was noticed in all the six muscle specimens of these three patients (Table 2).

A large nerve made up of five bundles, included in the same biceps muscle, showed feeble activity of the nerve fibres in SDH preparations (Fig. 9d), but clearer presence of oxidative enzymes within the fibres in NADH preparations (Fig. 9e).

The quadriceps muscle from the same patient showed a few relatively well-preserved fascicles, and a few ill-defined groups of small fibres (Fig. 10a) in a fibro-fatty matrix. There was an apparent preponderance of type I fibres as seen in both SDH and NADH preparations, with a ratio of type I:type II of $4: 1$ (Table 2). The feature of note was the presence in phosphorylase preparations of a large number of dark staining - that is, apparently type II fibres - so that the ratio here of type I:intermediate:type II fibres was $1 \cdot 1: 1: 3 \cdot 1$ (Table 2). This feature was seen in respect of large, small, and normal-sized fibres. It indicated that the same fibres manifested activity of both oxidative and glycolytic enzymes, and not that there was a true preponderance of either of the two main types of fibres. Fibre size or variation with a range of 26 to $98 \mu$ was agaip observed (Table 2). One unusually large type $\vec{\nabla} \overrightarrow{\mathrm{c}}$ fibre (diameter of $170 \mu$ ) is illustrated in Fig. 10잉응 which also shows a group of relatively sma type I fibres of 50-60 $\mu$ (compare with Fig. 96. $>$ A rather vague group atrophy was observed one or two spots. These features contrasted wi the extremely thin 'ill-developed' fibres of t other quadriceps muscle of this patient biopsied earlier (Fig. 1, Table 1).

\section{COMMENT}

On the basis of the initial histological examina- $\frac{\perp}{\varnothing}$ tion alone, the most impressive change in the $\overrightarrow{\overrightarrow{0}}$ muscle biopsy specimens was the presence in 3 nearly two-thirds of our cases with AMC of extremely thin muscle fibres embedded irregularly in fibrous tissue which formed the greater part of the specimen. This was irrespective of the age of the patient. While the smallness of 3 fibre size was suggestive of denervation, the absence of grouping, except in three of the $10 \frac{\circ}{3}$ muscles (cases $6,10,12$ ) and the presence of 0 connective tissue actually among the fibres, were features against a denervation atrophy. The fibres appeared smooth and faint and crossstriations were apparent only with PTAH o staining. The overall impression created was 0 that of ill-formed muscle fibres.

The mechanism of this lack of development is not clear, but failure or retardation in develop- $\stackrel{0}{?}$ 
ment of musculature in utero is one possibility and lack of neurogenic stimulus or trophic impulse is another. The former appears a plausible mechanism, since it is known that nerves are not concerned with organizing primary muscle formation (Hamilton, Boyd, and Mossman, 1962). There is at least evidence in experimental animals that muscles will develop in limbs devoid of innervation (Hamburger, 1939).

It is relevant at this juncture to recapitulate a few salient facts regarding the development of musculature in the human foetus. These observations have been drawn mainly from Hamilton et al. (1962). In general, it appears that the limb muscles of most tetrapods are not derived from the myotomes but from the limb buds. With the exception of nerve fibres, Schwann cells, melanoblasts, and possibly the endothelium of blood vessels and lymphatics, all the subepidermal cellular elements of the limb including those of its muscles are now considered to take their origin in situ from the mesenchyme of the embryonic buds. The intrinsic muscles of the limbs also differentiate in situ.

Branches of the nerves supplying the segments corresponding to those of the limb-bud reach its base. As the bud elongates to form a limb, they extend into it. The muscles split into a ventral or limb-flexor group and a dorsal or limb-extensor group. The nerves of a limb are likewise divided into anterior or posterior divisions supplying flexor and extensor muscles respectively. Thus the muscles in the adult limb have a fairly consistent segmental innervation.

As development of myoblasts proceeds, they become elongated and increase in girth, so that in the human embryo of only 5-8 mm CR-length myofibrillae can be identified. From being uninucleate, the myoblasts become multinucleate and, when they are about $1 \mu$ in diameter, cross-striations appear. They assume a tubular structure and the myotubes are converted into muscle fibres. In the last trimester of gestation the central nuclei migrate to the margin of the fibres which increase in girth by multiplication of their myofibrillae.

Striated muscles are said to grow from recruitment of new myoblasts from the adjacent mesenchyma, by divisions of early myoblasts before fibrils have formed and by the increase in size of the individual fibres. Postnatal growth seems to be entirely due to enlargement of pre- existing muscle fibres. The tendons of muscles seem to develop independently of the muscular tissue, and are secondarily linked to it by the connective tissue of the perimysium. Hence, as Hamilton et al. (1962) conclude, the contractile and the connective tissue elements in a muscle constitute a composite organ rather than a single uniform tissue.

Extrapolating these embryological observations to our histological findings on muscles in AMC, it seems likely that in this condition the development of the limb-buds and even of individual muscle fibres may be adequate, but the total number of fibres developed and present at birth may be grossly inadequate. In other words, the recruitment of the myoblasts from the adjacent mesenchyme appears defective. The net result may be a lack of differentiation of the mesenchymal mass into individually recognizable muscles. The mesenchyme seems to have differentiated preferentially and excessively towards the formation of collagenous fibrous tissue. Since no new muscle fibres can be expected to develop after birth (vide supra), whatever postnatal growth of muscles occurs in patients with AMC would probably be the result of hypertrophy of the pre-existing fibres. Where the process is not very severe and at least a fair complement of muscle fibres is present, a reasonable development of muscle bulk may be expected and perhaps boosted by the stimulus of physiotherapy and exercises (see discussion by Bharucha et al. (1972)).

Our surgical colleague at the Children's Orthopaedic Hospital in Bombay, who carries out successive operations to correct the joint deformities in such children, occasionally reports the development of grossly detectable muscle planes where at earlier operations no muscle tissue could be visualized at all and the deep fascia and 'muscles' may even have looked like one fibrous mass (Mullaferoze, 1970).

While nerve supply is not imperative to the initial development of muscle, innervation nevertheless seems essential for the further growth of muscle fibres. In the pig, for instance, while the muscle differentiates before nerve connections are established, the latter are necessary for full maturation, as shown originally by Bardeen (1900). In the muscle of the human foetus nerve fibres are recognizable at about 10 weeks' gestation and terminal sprouts make contact with muscle fibres by the 11 th week, as demonstrated by Tello (1922) and Cuajunco 
(1942). However, it is not until between the 14th and 24th weeks that the motor endplates start developing and they probably reach their full maturity a little later than that. This would mean that adequate muscle fibre growth from the 4th to the 6 th month can take place only under the stimulus of nerve supply.

During early embryogenesis, till about the 20 th week in the human foetus, there is no clear histochemical differentiation of muscle fibres into different types (Dubowitz, 1966). An essentially uniform pattern with type II fibres predominating develops by the 30th week (Fenichel, 1966). During the last few weeks of gestation, there is a shift towards the normal checkerboard pattern of type I and type II fibres (Dubowitz, 1968).

Hence, in AMC, a possible mechanism would be normal initial development of muscle fibres from myoblasts, but arrest or retardation of their further development from about the 11th week onwards on account of lack of neural stimulus. This could result from failure of development or early degeneration of anterior horn cells in the spinal cord, as in fact has been observed in a certain proportion of cases by a number of workers (reviewed by Bharucha et al., 1972).

It is equally conceivable that due to a disorder early in embryonic life both the myoblast and the neuroblast are retarded in their further differentiation into the muscle fibre and the anterior horn cell respectively. It may be recalled that the maturation of the anterior horn cells from the neuroblasts of the basal lamina of the primitive spinal cord takes place during about the second month of intrauterine life or before the embryo reaches the $30 \mathrm{~mm}$ stage (Hamilton et al., 1962). Hence in some cases of AMC primary muscle fibre development may suffer more, while in others impairment in further growth of muscle fibre under a neural stimulus may be the predominant defect.

The evidence from the repeat biopsy specimens from three of the cases, collected mainly for histochemical examination, was difficult to evaluate definitively on account of the presence of very few fibres in three of the six specimens obtained. However, four interesting points emerged. In case 3, where initially a normal muscle had been sampled, clinically weak muscles from arm and forearm (Table 2) showed a histochemically normal checkerboard pattern and normal ratio of fibre size throughout both specimens, suggesting disuse atrophy, probably resulting from limitation of movement $z$ at the joints concerned. Identical disuse atrophy was observed by us with immobilized limbs (Patel, Razzak, and Dastur, 1969).

In case 4 , while the very few generally small 0 and scattered fibres did not permit any histological diagnosis, the groups of clustered nuclei $\stackrel{\circ}{=}$ in $\mathrm{H}$ and $\mathrm{E}$ stained frozen sections, with a stray muscle fibre in two or three groups, perhaps:indicated a loss of preformed fibres. The total $\stackrel{\overrightarrow{\bar{s}}}{\rightarrow}$ number of fibres in both specimens was too few to stress the relative preponderance of type $I$ 음 fibres, but a depletion of type II fibres would $\frac{\bar{\omega}}{\vec{D}}$ point to denervation (Engel and Brooke, 1965). $\stackrel{\mathbb{2}}{\varnothing}$ In four of Fenichel's (1969) cases of 'arthrogryposis' with brain malformation, there was a $\vec{\circ}$ wider spectrum of fibre size with largest fibres of $\overrightarrow{-}$ type II, but both fibre types in equal proportions. $\vec{\omega}$ The rounding of fibres observed was taken merely as a feature of the lack of support of these scattered fibres which were generally of floating about in the fibro-fatty tissue as happens + in muscular dystrophy, but this rounding alor was not taken to be evidence for dystrophy i this case.

The two muscles of case 14 were differe $\square$ from one another, but while the more affecteg 0 specimen of $\mathrm{M}$. biceps brachii contained relog क tively few fibres, they both shared the commati $\overrightarrow{0}$ feature of a few huge hypertrophied fibres $N$ (Table 2). This was in line with our earlier o expectations. Equally interesting was the presence in the same fibres, in the less affected quadriceps muscle, of activity for both oxidative enzymes and phosphorylase. This appeared possible if the muscle fibres were still in an undeveloped state. However, this muscle contrasted with the opposite quadriceps biopsied $2 \frac{1}{2}$ years earlier, in that excessively thin illdeveloped fibres were not seen any more and indicated that the muscle had in fact 'grown' in a small but appreciable manner.

The presence of large numbers of well-formed muscle spindles and nerves in all the six repeat biopsy specimens, reflected at least the integrity of peripheral nerves and indirectly suggested that at least a proportion of the spinal motorneurones had developed and made contact with the appropriate muscles.

Either on the basis of dysplastic muscle fibres or of ill-formed anterior horn cells, proliferation of fibrous tissue in the muscle may be expected. Deformities of the limbs and contractures at the 
joints, which constitute the hallmark of the clinical picture of AMC, could naturally be expected to result from excessive fibrosis of groups of muscles and due to inadequacy of muscle tissue available to produce movements at the joints. According to Badgley (1943), if the limb-bud rotation occurring normally in the third foetal month fails due to embryonic arrest of muscle development, then the joints do not develop and articular surfaces do not form. He postulates this mechanism for the production of congenital dysplasias of the hip.

Another feature to be expected with either of the alternative pathogenetic mechanisms would be a lack of progression of the disease after birth, since there is no evidence, in our material or of others, of either clinical, electromyographic, or pathological progression. Moreover, electrical evidence of regeneration was generally lacking, as was the case with all of our patients who showed denervation potentials (Bharucha et al., 1972). It is also significant that two of our 10 cases with atrophic looking fibres revealed a normal pattern on EMG. Thus there is no similarity here with either the infantile or the juvenile types of spinal muscular atrophy.

One also wonders if there is a truly dystrophic form of AMC. The two original cases of Banker et al. (1957) and some of those of Pearson and Fowler (1963) show how congenital muscular dystrophy may occasionally be accompanied by fibrotic contractures similar to those of AMC. Such observations prove some of the joint deformities to be non-specific, but do not necessarily establish a myopathic form of AMC. This disease is probably not the common denominator of a variety of pathogenetic processes, but the result of one or two onlynamely, an embryonal lack of development of spinal motor neurones and/or deficient histogenesis of muscle per se. The two cases in our own series which we have tentatively classified as 'myopathic' did not satisfy all the histological criteria of a muscular dystrophy and did not show an elevation of serum CPK.

Thus the overall impression on histological and histochemical examination, including the follow-up study, was that lack of development of muscle was responsible for AMC in the majority of our cases. In a small proportion (about a third) denervation atrophy could have been the main or an additional factor.

\section{REFERENCES}

Badgley, C. E. (1943). Correlation of clinical and anatomical facts leading to a conception of the etiology of congenital hip dysplasias. Journal of Bone and Joint Surgery, 25, 503523.

Banker, B. Q., Victor, M., and Adams, R. D. (1957). Arthrogryposis multiplex due to congenital muscular dystrophy. Brain, 80, 319-334.

Bardeen, C. R. (1900). The development of the musculature of the body wall in the pig, including its histogenesis and its relations to the myotomes and to the skeletal and nervous apparatus. Johns Hopkins Hospital Report, 9, 367-400.

Bharucha, E. P., Pandya, S. S., and Dastur, D. K. (1972). Arthrogryposis multiplex congenita: I: Clinical and electromyographic aspects. Journal of Neurology, Neurosurgery, and Psychiatry, 35, 425-434.

Cuajunco, F. (1942). Development of the human motor end plate. Contributions to Embryology, 30, 127-152.

Drachman, D. B., and Coulombre, A. J. (1962). Experimental clubfoot and arthrogryposis multiplex congenita. Lancet, 2, 523-526.

Dubowitz, V. (1966). Enzyme histochemistry of skeletal muscle. III. Neurogenic muscular atrophies. Journal of Neurology, Neurosurgery, and Psychiatry, 29, 23-28.

Dubowitz, V. (1968). 'Developing and diseased muscle'. A histochemical study, pp. 61-64. SIMP Research Monograph 2. Heinemann: London.

Engel, W. K., and Brooke, M. H. (1966). Muscle biopsy as a clinical diagnostic aid. In Neurological Diagnostic Techniques, pp. 90-146. Edited by W. S. Fields. Thomas: Springfield, Ill.

Fenichel, G. M. (1966). A histochemical study of developing human skeletal muscle. Neurology (Minneap.), 16, 741745.

Fenichel, G. M. (1969). Cerebral influence on muscle fibre typing. The effect of fetal immobilization. Archives of Neurology, 20, 644-649.

Frischknecht, W., Bianchi, L., and Pilleri, G. (1960). Familiäre Arthrogryposis multiplex congenita: Neuroarthro-myodysplasia congenita. Helvetica Paediatrica Acta, 15, 259-279.

Hamburger, V. (1939). The development and innervation of transplanted limb primordia of chick embryos. Journal of Experimental Zoology, 80, 347-389.

Hamilton, W. J., Boyd, J. D., and Mossman, H. W. (1962). Human Embryology. 3rd edition. Heffer: Cambridge.

Hillman, J. W., and Johnson, J. T. H. (1955). Arthrogryposis multiplex congenita in twins. Journal of Bone and Joint Surgery, 34-A, 211-214.

Howard, R. (1908). A case of congenital defect of the muscular system (dystrophia muscularis congenita) and its association with congenital talipes equino-varus. Proceedings of the Royal Society of Medicine, Vol. 1, pt 3, 157-166.

Hughes, B. P. (1962). A method for the estimation of serum creatine kinase and adolase activity in normal and pathological sera. Clinica Chimica Acta, 7, 597-603.

Kite, J. H. (1955). Arthrogryposis multiplex congenita: review of 54 cases. Southern Medical Journal, 48, 11411146.

Middleton, D. S. (1934). Studies on prenatal lesions of striated muscle as a cause of congenital deformity. Edinburgh Medical Journal, 41, 401-442.

Mullaferoze, P. (1970). Personal communications.

Padykula, H. A., and Herman, E. (1955). The specificity of the histochemical method for adenosine triphosphatase. Journal of Histochemistry and Cytochemistry, 3, 170-195.

Patel, A. N., Razzak, Z. A., and Dastur, D. K. (1969). Disuse atrophy of human skeletal muscles. Archives of Neurology, 20, 413-421. 
Pearson, C. M., and Fowler, W. G., Jr. (1963). Hereditary non-progressive muscular dystrophy inducing arthrogryposis syndromes. Brain, 86, 75-88.

Razzak, Z. A. (1970). Studies in muscular and neuromuscular disorders; Part C; Muscle histochemistry and histology. M.Sc. Thesis of the University of Bombay.

Rossi, E. (1947). Le syndrome arthromyodysplasique congénital. Helvetica Paediatrica Acta, 2, 82-97.

Sheldon, W. (1932). Amyoplasia congenita. Multiple congenital articular rigidity: arthrogryposis multiplex congenita. Archives of Diseases in Childhood, 7, 117-136.

Swinyard, C. A., and Mayer, V. (1963). Multiple congenital contractures. I: Public health considerations of arthrogryposis multiplex congenita, Journal of the American Medical Association, 183, 23-27.

Tello, J. F. (1922). Die Entstehung der motorischen und sensiblen Nervenendigungen. 1. In dem lokomotorischen System der höheren Wirbeltiere. Muskuläre Histogenese. Zeitschrift für Anatomie und Entwicklungsgeschichte, 64, o 384-440. 